\title{
Unusual Clinical Presentations in a Patient with Novel ADCK3 Variants
}

\author{
Eui Ra Huh, MD, Hyunjoo Lee, MD, Young-Mock Lee, MD \\ Department of Pediatrics, Yonsei University College of Medicine, Seoul, Korea
}

Received: October 14, 2020

Accepted: October 30, 2020

Corresponding author:

Young-Mock Lee, MD

Department of Pediatrics, Gangnam

Severance Hospital, Yonsei

University College of Medicine, 211

Eonju-ro, Gangnam-gu, Seoul

06273, Korea

Tel: +82-2-2019-3354

Fax: +82-2-3461-9473

E-mail:ymleemd@yuhs.ac
The AarF domain containing kinase 3 (ADCK3) is a mitochondrial protein required for coenzyme $\mathrm{Q}(\mathrm{CoQ})$ biosynthesis. Loss of function mutations in the $A D C K 3$ gene cause coenzyme Q10 (CoQ10) deficiency (\#MIM 612016), resulting in mitochondrial dysfunction. Cerebella ataxia is its most common phenotype [1]. However, acute epileptic encephalopathy with stroke-like episodes has been reported in few cases [2]. Here, we report the case of a patient with novel $A D C K 3$ mutations who presented with a homonymous hemianopsia, stoke like brain lesions in brain magnetic resonance imaging (MRI).

A 3-year-old girl presented with exercise intolerance, gait disturbance and occasional dysarthria following normal birth and development. Serum creatinine kinase and lactate dehydrogenase were elevated at $964 \mathrm{U} / \mathrm{L}$ ( 21 to 215) and $3,427 \mathrm{IU} / \mathrm{L}$ (255 to 455$)$. And serum lactate was high at $8.3 \mathrm{mmol} / \mathrm{L}$ (0.5 to 1.6$)$. We observed ragged red fibers on muscle biopsy and identified mitochondrial respiratory chain complex 1 deficiency from biochemical tests. She was diagnosed with a mitochondrial disorder. The first generalized tonic seizure occurred at the age of 6 years and was administered anti-epileptic drugs. At 14 years of age, she presented with stroke-like episodes with severe headache, nausea, dysarthria, and left-sided hemianopsia. However, her brain MRI findings were normal. Electroenceph- alogram (EEG) showed frequent epileptic discharges in the right occipital area. Those acute symptoms improved after 7 days of conservative management to reduce cerebral edema with hyperosmolar agent (mannitol) and high-dose corticosteroids. She reported another stroke-like episode with similar symptoms at the age of 18 years. In addition, she experienced sudden left-sided homonymous hemianopsia and dysarthria concomitant with ataxic gait and exercise intolerance. We detected generalized tonic seizure during her hospitalization. Repeated brain MRI showed focal areas of high-signal intensity in the right occipital lobe and dentate nucleus. Nonetheless, brain magnetic resonance angiography showed no abnormal vascular lesion. While there were frequent sharp wave discharges from both occipital areas in EEG, a visual field examination showed ipsilateral left hemianopsia.

Whole-exome sequencing was performed for accurate diagnosis because no significant variant was detected in the previous whole mitochondrial genome test. In this study, the compound heterozygous mutations were identified, namely c. 655+ $1 \mathrm{G}>\mathrm{A}$ and c.1015G > A (p.Ala339Thr) in the ADCK3 gene (NM_020247.4 and NP_06463 2.2). According to the American College of Medical Genetics and Genomics guidelines, c.655+ $1 \mathrm{G}>\mathrm{A}$ is a novel pathogenic variant and c.1015G $>\mathrm{A}$ is a likely pathogenic variant which has been

Copyright (C) 2021 Korean Child Neurology Society

This is an Open Access article distributed under the terms of the Creative Commons Attribution Non-Commercial License (http://creativecommons.org/licenses/by-nc/4.0/) which permits unrestricted non-commercial use, distribution, and reproduction in any medium, provided the original work is properly cited. 

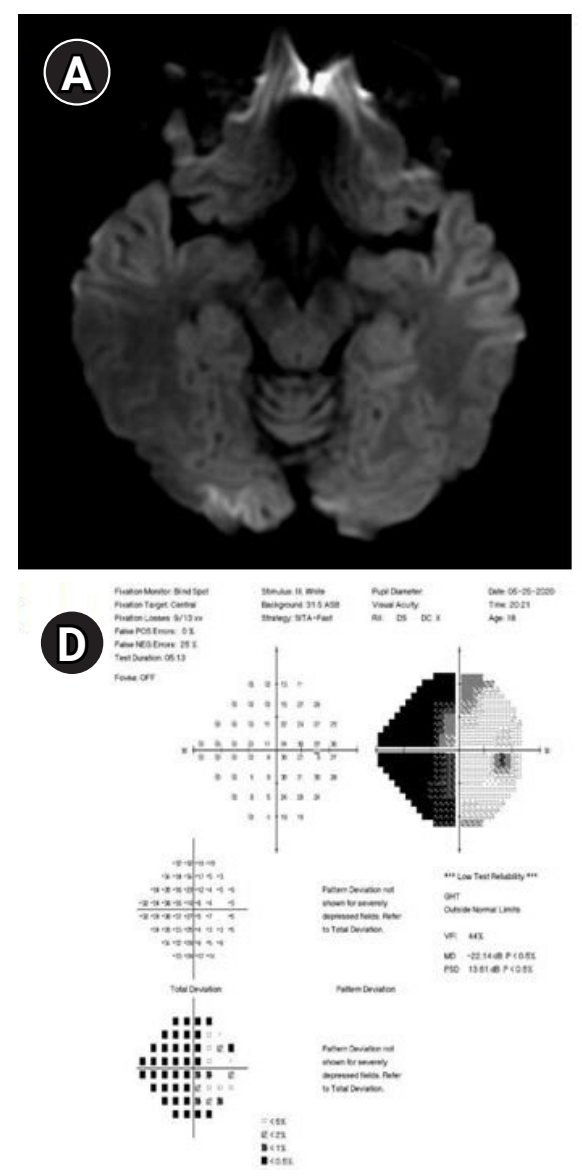
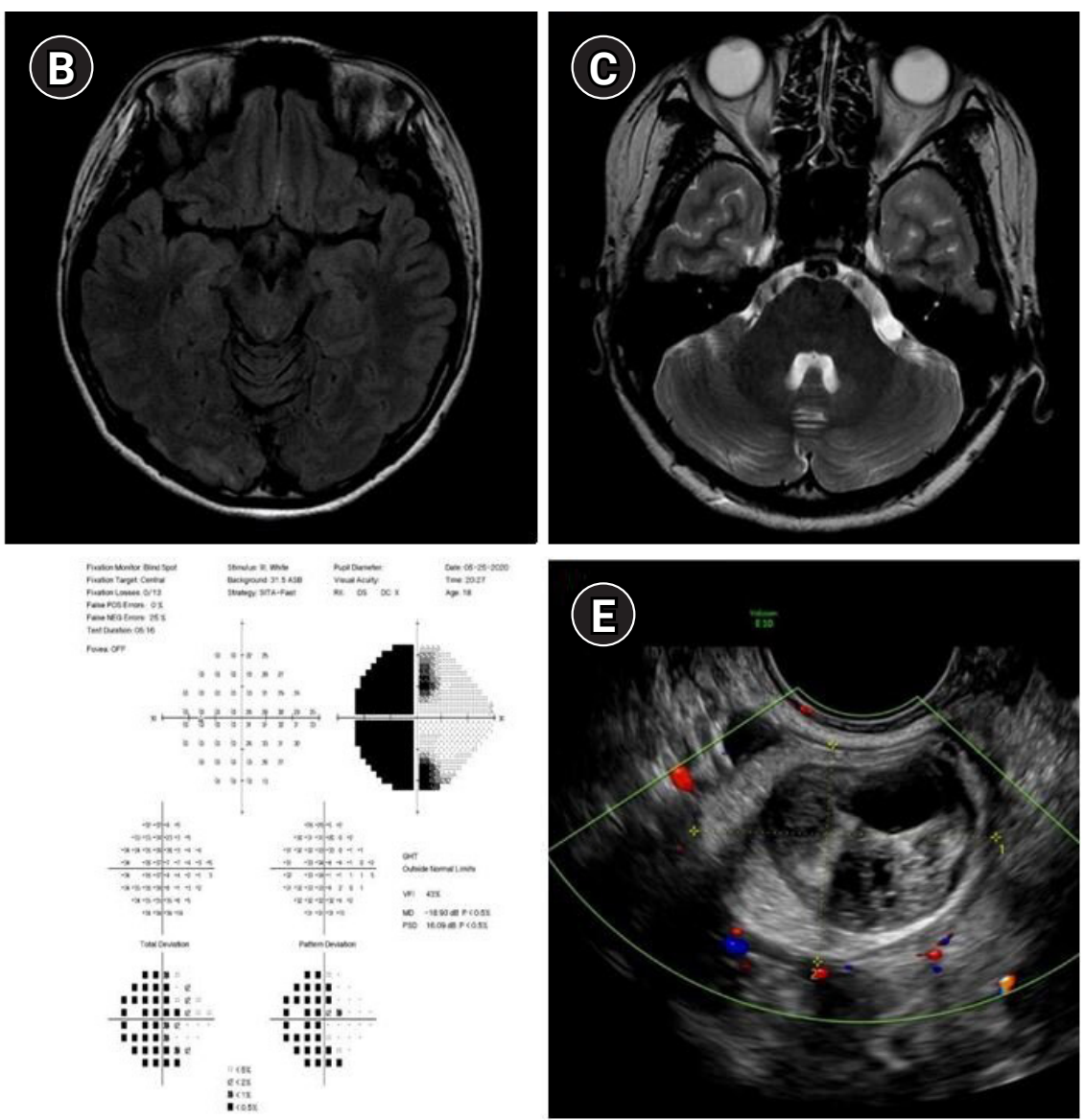

Fig. 1. A patient with coenzyme 010 deficiency diagnosed with compound heterozygous variants in the AarF domain containing kinase 3 (ADCK3) gene: $(A)$ a focal abnormal lesion with restricted diffusion at right occipital lobe; $(B)$ increased signal intensity at right occipital lobe on T2 weighted image; (C) increased signal intensity at dentate nucleus on T2 weighted image; (D) homonymous hemianopia; and (E) ovarian teratoma.

reported in a patient with a homozygote mutation [3]. The variants were confirmed by Sanger sequencing in the proband and both parents and considering the autosomal recessive inheritance of the $A D C K 3$ gene, it is likely that the asymptomatic parents were carriers. After genetic diagnosis, she has been taking CoQ10 supplements with $400 \mathrm{mg}$ daily.

Hemianopia and stoke-like lesions are common symptoms of mitochondrial encephalopathy, lactic acidosis, and stroke-like episodes (MELAS). In a patient suspected of mitochondrial cytopathy with stroke-like lesions on brain MRI without pathogenic variants related to MELAS, it is recommended to consider testing for mitochondrial disorders caused by nuclear DNA mutations, including the $A D C K 3$ gene.

$A D C K 3$-related CoQ10 deficiency is a rare disease. Furthermore, the phenotype is neither classified systematically nor well known. Patients with $A D C K 3$ mutations usually present with progressive cerebellar ataxia, exercise intolerance, and high-signal intensity of the dentate nucleus. However, they rarely report cardio- myopathy and nephrotic syndrome. In addition, her abdominal radiography revealed ovarian calcification and a mature ovarian teratoma was confirmed by ultrasound findings incidentally (Fig. 1). There are no reports of a definite association with the $A D C K 3$ gene, which causes the deficiency of CoQ10, which plays an important role in antioxidant.

To our knowledge, this is the first report of a patient with novel compound heterozygous $A D C K 3$ variants in Korea who showed signs of ataxia, exercise intolerance, homonymous hemianopsia, dysarthria, and teratoma. This report aims to expand the understanding of the genotype-phenotype spectrum associated with $A D C K 3$-related CoQ10 deficiency.

This study was approved by the Institutional Review Board of Gangnam Severance Hospital (approval number: 3-2020-0393). Informed consent was waived by the board.

\section{Conflicts of interest}


No potential conflict of interest relevant to this article was reported.

\section{ORCID}

Eui Ra Huh, https://orcid.org/0000-0002-6998-981X

Young-Mock Lee, https://orcid.org/0000-0002-5838-249X

\section{Author contribution}

Conceptualization: ERH, HL, and YML. Data curation: ERH, HL, and YML. Formal analysis: ERH, HL, and YML. Funding acquisition: ERH, HL, and YML. Methodology: ERH, HL, and YML. Project administration: ERH, HL, and YML. Visualization: ERH, HL, and YML. Writing-original draft: ERH. Writing-review \& editing: ERH, HL, and YML.

\section{References}

1. Emmanuele V, Lopez LC, Berardo A, Naini A, Tadesse S, Wen B, et al. Heterogeneity of coenzyme Q10 deficiency: patient study and literature review. Arch Neurol 2012;69:978-83.

2. Hikmat O, Tzoulis C, Knappskog PM, Johansson S, Boman H, Sztromwasser P, et al. ADCK3 mutations with epilepsy, strokelike episodes and ataxia: a POLG mimic? Eur J Neurol 2016;23: 1188-94.

3. Cotta A, Alston CL, Baptista-Junior S, Paim JF, Carvalho E, Navarro $\mathrm{MM}$, et al. Early-onset coenzyme Q10 deficiency associated with ataxia and respiratory chain dysfunction due to novel pathogenic COQ8A variants, including a large intragenic deletion.JIMD Rep 2020;54:45-53. 